\title{
QUANTUM MOLECULAR DYNAMICS SIMULATIONS OF CONJUGATED POLYMERS
}

\author{
A. M. Almeida, Marta M. D. Ramos ${ }^{*}$, A. M. Cadilhe \\ Departamento de Física, Universidade do Minho, Largo do Paço, \\ 4700-320 Braga, Portugal
}

\begin{abstract}
The softness of conjugated polymers leads to strong coupling between polymer's electrons and lattice vibrations. Therefore, it is necessary to perform quantum molecular dynamics computer simulations in order to study their electronic and optical properties at molecular level. We have used self-consistent molecular dynamics calculations with inter-atomic forces evaluated from quantum mechanical calculations at the CNDO (complete neglect of differential overlap) level to discuss some of the issues relating to the electronic processes involved in polydiacetylene (PDA) and poly(p-phenylene vinylene) (PPV). Specifically addressed are the charge induced structural changes of the polymer chains and the intra-molecular charge mobility. The change in the chemical
\end{abstract}

* Corresponding Author: Tel:+351 253 604330; Fax:+351 253678 981; E-mail:marta@ fisica.uminho.pt 
potential of individual polymer strands at zero temperature is also discussed. Our results suggest a geometrical distortion in the bond length distribution relative to the uncharged chains which is accompanied by changes in atomic charges at the distortion site. The charge carrier mobility is predicted to depend on the strength of the electric field, in accordance with experiments.

Keywords: Quantum molecular dynamics, conjugated polymers, charge-induced defects.

\section{Introduction}

Conjugated polymers are organic semiconductors with $\pi$ molecular orbitals delocalized along the polymer chain. Their unusual electrical and optical properties makes them ideally suitable to be used as active components of electronic [1] and optoelectronic [2] devices. The development and optimization of these organic materials requires computer simulations to provide fundamental understanding of the electronic processes at molecular level.

What is required to deal with systems such as conjugated polymers, where atomic positions and electronic structure are coupled problems, is a simulation scheme that finds adiabatic (BornOppenheimer) electronic distribution appropriated to each atomic configuration, from which the forces on the atoms are calculated, and to use the interatomic forces to move the atoms according to newtonian equations of motion. The electronic structure calculations may be performed using quantum mechanical techniques such as pseudopotentials [3], molecular orbital theories at ab initio

or semi-empirical level [4] and density functional theory [5]. All these self-consistent methods attempt, at some level of approximation, to solve the Schrödinger equation for the entire system. The type of method preferred depends on the property to be studied and, mainly, on the degree of accuracy required and computer power available. 
The present work examines the charge injection and transport in polydiacetylene (PDA) and poly(pphenylene vinylene) (PPV) chains of different length. The results were obtained by molecular dynamics combined with a semi-empirical application of molecular orbital approach.

\section{Theoretical method}

The approach we adopt combines self-consistent quantum mechanical method and molecular dynamics. While the use of a highly accurate parameter free quantum mechanical method is desirable, it becomes computationally prohibitive for large systems such as long polymer chains. Therefore, we have used an approximate molecular orbital method, called CNDO (Complete Neglect of Differential Overlap), that has been sucessfully tested and validated in many thousands of previous calculations. Within a minimal-basis representation the valence electron wavefunctions are considered in the LCAO (Linear Combination of Atomic Orbital) approximation. The gradients of the total self-consistent energy, evaluated at the given atom positions, yield the interatomic forces. A molecular dynamical method is used in parallel to move the atoms according to those forces. Within these simulations the atoms of an initial structural configuration move, within the Born-Oppenheimer approximation, on the potential energy surface obtained directly from the electronic structure calculation.

\section{Results and discussion}

\subsection{Uncharged molecules}

The ground state geometries of uncharged PDA and PPV molecules were found by examining straight planar molecules of $\mathrm{C}_{4 \mathrm{n}+2} \mathrm{H}_{2 \mathrm{n}+4}$ and $\mathrm{C}_{8 \mathrm{n}} \mathrm{H}_{6 \mathrm{n}+2}$, respectively, where $\mathrm{n}$ is the number of 
repeated units in each polymer chain. Polymer strands of different length were obtained by varying the number of units from 2 to 16.

\subsection{Charge-induced distortion of polymer molecules}

In order to study the charge-induced distortion in the polymer chain, it is helpful to define the dimerisation parameter by

$$
d_{n}=(-1)^{n}\left(b_{n, n+1}-b_{n-1, n}\right)
$$

where $b_{n, n+1}$ is the bond length between atoms $n$ and $n+1$. This parameter represents the degree of dimerisation along the polymer chain.

The geometrical distortion in the bond length distribution of an uncharged PDA chain corresponding to the injection of an electron is shown in Fig. 1. The resulting distortion pattern show two main features. First, there are two different degrees of dimerisation along the uncharged polymer chain corresponding to the difference between single and triple bond lengths within each repeated unit and the length difference between the double bond linking two diacetylene units and the adjacent single bonds. Second, the charge-induced distortion leads to a decrease in dimerisation at the central region of the polymer chain. Our calculations predict no real difference between the dimerisation pattern of both positive and negative charged PDA.

The distortion pattern for uncharged PPV shows three different types dimerisation, since there are two inequivalent $\mathrm{C}-\mathrm{C}$ and $\mathrm{C}=\mathrm{C}$ bonds [6]. The width of the distortion in single charged PDA is rather greater than that of PPV [ 6]. 


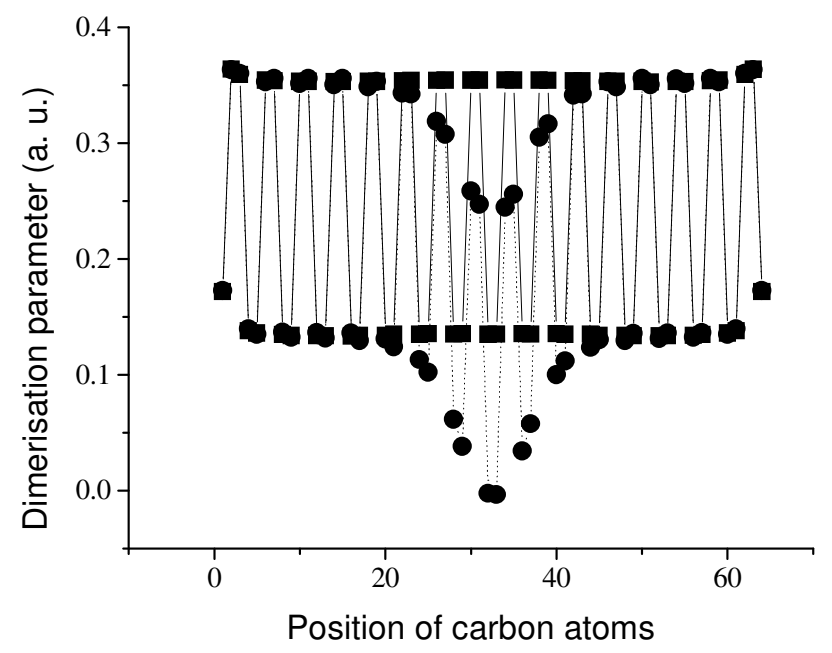

Fig. 1. Dimerisation patterns in a PDA chain with 16 repeated units: (a) neutral (squares), (b) after injection of one electron (circles). The curves are simply a guide to the eye.

\subsection{Charge rearrangement among the polymer atoms}

The charge distribution associated with charged PPV and PDA chains shows two important features. Firstly, there is a good deal of charge stored on the hydrogen atoms, rather than the entire injected charge resting on the carbon atoms of the polymer chain. The amount of charge stored on both type of atoms depends on the polymer geometry (see Table 1).

\section{Table 1}

Charge stored in hydrogen and carbon atoms of negative charged PPV and PDA chains with 16 repeated units.

\begin{tabular}{|c|c|c|}
\hline \multirow{2}{*}{ Polymer } & \multicolumn{2}{|c|}{ Charge (\%) } \\
\cline { 2 - 3 } & Carbon atoms & Hydrogen atoms \\
\hline PPV & 55 & 45 \\
\hline PDA & 72 & 28 \\
\hline
\end{tabular}


Secondly, the charge stored on each carbon atom alternates along both polymer chains on the same region as the distortion occurs $[6,7,8]$. Besides, the charge stored on the hydrogen atoms is mainly localized at distortion site and is does not display any alternation.

\subsection{Chemical potential at zero temperature}

The chemical potential of a thermodynamic system is defined by:

$$
\phi=\left(\frac{\partial E}{\partial N}\right)_{T}-T\left(\frac{\partial S}{\partial N}\right)_{T}
$$

where $E$ is the internal energy, $S$ is the entropy, $T$ is the thermodynamic temperature and $N$ represents the number of electrons of the system.

The zero temperature limit of the chemical potential can be obtained from the curve internal energy versus molecular charge. It corresponds to the slope of this curve when no electron is added or removed from the uncharged system. The dependence of the internal energy, $E$, of a PDA chain with 7 repeated units on net charge, $Q$, is shown in Fig.2. By assuming the following parabolic fit to this curve

$$
E=\left(-224.7+0.166 Q+0.069 Q^{2}\right)(\text { Hartrees })
$$

we obtain

$$
\phi=\left(\frac{\partial E(Q)}{\partial Q}\right)_{Q=0}=0.166 \text { Hartrees }
$$

in agreement whith the value calculated using three point finite-difference method, i.e. the arithmetic average of electron affinity (energy gain on adding an electron) and ionization potential 
(energy to remove an electron) [6]. The change in the chemical potential of this PDA chain associated with a change in its number of electrons is essentially

$$
\phi(Q)=\phi(Q=0)+\Delta \phi=(0.166+0.069 Q)(\text { Hartrees })
$$

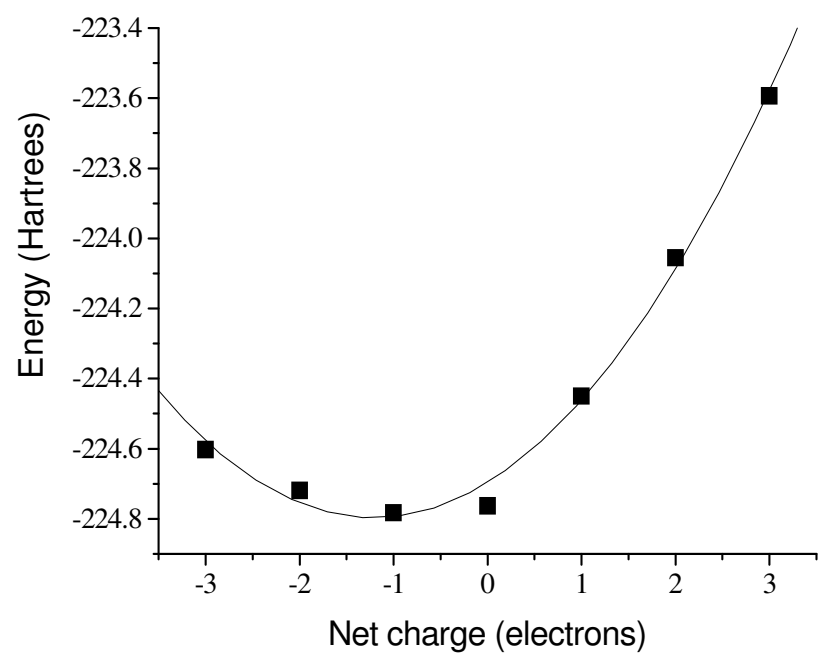

Fig. 2. Dependence of internal energy of PDA chain with 7 repeated units on net charge. The marks on the curve indicate the data points that were calculated explicitly.

\subsection{Charge carrier drift}

An uniform electric field along the polymer chain is responsible for the drift on the charge-induced defects. Our results suggest that the intra-molecular mobility of PDA and PPV is a function of the electric field. Nevertheless, the threshold for charge carrier mobility depends on the polymer structure (see Table 2). 


\section{Table 2}

Electric field threshold for intra-molecular charge carrier mobility in PDA and PPV chains with 16 repeated units.

\begin{tabular}{|c|c|}
\hline Polymer & Electric field threshold $\left(\times 10^{8} \mathrm{~V} / \mathrm{m}\right)$ \\
\hline PDA & $2.35 \pm 0.15$ \\
\hline PPV & $1.65 \pm 0.15$ \\
\hline
\end{tabular}

\section{Conclusions}

Computer simulations may play an important role to achieve a detailed understanding of structureproperties correlations in conjugated polymers at molecular level. We have discussed our own contribution, using a quantum molecular dynamics approach. It has been shown that single charges injected into conjugated polymer chains give rise to charge-induced defects and charge rearrangement of the polymer atoms at the distortion site. When an electric field is applied, the charge carrier moves along the polymer chain if the applied field is greater than a certain value. The knowledge of the effect of chain length and molecular charge on the chemical potential of individual chains that we have gained through our calculations will allow us to predict when intermolecular charge transfer within the polymer film is not possible.

We believe that computer simulations of the type reported here have the ability to provide an insight into the physics underlying the intramolecular charge transport in conjugated polymers in a more effective way than experiments. 


\section{References}

[1] J. H. Burroughes, C. A. Jones, R. H. Friend, Nature 335 (1988) 137.

[2] R. H. Friend, R. W. Gymer, A. B. Holmes, J. H. Burroughes, R. N. Marks, C. Taliani, D. D. C. Bradley, D. A. Dos Santos, J. L. Brédas, M. Lögdlung and W. R. Salaneck, Nature 397 (1999) 121.

[3] J. H. Burroughes, D. C. Bradley, A. R. Brown, R. N. Marks, K. Mackay, R. H. Friend, P. L. Burns and A. B. Holmes, J. Chem. Soc. Faraday Trans. 86 (1990) 1221.

[4] J. A. Pople, D. L. Beveridge, ApproximateMolecular Orbital Theory, McGraw-Hill, New York, 1970.

[5] R.G. Parr, W. Yang, Density-Functional Theory of Atoms and Molecules, Oxford University Press, Oxford, 1989.

[6] M. M. D. Ramos, A. M. Almeida, J. P. M. Carneiro, A. M. Stoneham, Le Vide: science, technique et applications, in print (2001).

[7] M. M. D. Ramos, A. M. Almeida, Vacuum, in print (2001).

[8] A. M. Almeida, M. M. D. Ramos, Synthetic Metals 7718 (2001) 165. 